\title{
A INFLUÊNCIA DA ANSIEDADE NA RESPOSTA DO PACIENTE NO PERÍODO PÓS-OPERATÓRIO. ${ }^{1}$
}

\section{THE INFLUENCE OF THE ANXIETY IN THE PATIENTS ANSWER IN THE POST OPERATIVE PERIOD}

\author{
Aparecida de Cassia Giani Peniche* \\ Vanda Maria Galvão Jouclas** \\ Eliane Corrêa Chaves*
}

PENICHE, A. de C. G. et al. A influência da ansiedade da resposta do paciente no período pós-operatório. Rev. Esc. Enf. USP, v. 33, n. 4, p. 391-403, dez. 1999.

\section{Frstumo}

O homem, ao se defrontar com a cirurgia e a anestesia, tem alterações hormonais provocadas pela ansiedade, moduladas pela avaliação cognitiva deste enfrentamento e acompanhadas de alterações fisiológicas perceptiveis. Os objetivos do presente estudo foram verificar a correlação entre o traço e o estado de ansiedade no periodo pré-operatóro, e a influência da ansiedade, na resposta do paciente, no periodo pós-operatório imediato, em sala de recuperação anestésica. A amostra foi composta por 33 pacientes cirúrgicos. Para a coleta de dados foram utilizados, além dos inventários auto-aplicáveis de traço-estado de ansiedade do paciente no periodo pré-operatório imediato, dois (2) formulários contendo parámetros de avaliação clínica dos pacientes. Os dados foram coletados nos períodos pré-operatório imediato e pós-operatório imediato. Os resultados mostraram uma correlação estatisticamente significante e positiva entre o traço e o estado de ansiedade, no periodo pré-operatório ou seja, os pacientes ao enfrentar o ato anestésico-cirúrgico, têm seu estado de ansiedade alterado, quando correlacionado ao seu traço de ansiedade e uma correlação não significante entre o estado de ansiedade, no préoperatório imediato com os parâmetros clínicos avaliados no periodo pós-operatório imediato, isto é, os pacientes não apresentaram alterações decorrentes do estado de ansiedade, no período pós-operatório imediato.

UNITERMOS:Ansiedade. Período pós-operatório. Período de recuperação da anestesia.

\section{W.}

The man confronting surgery and anesthesia, has hormonal alterations provoked by the anxiety, modulated by the cognitive evaluation done in the person that faces this situation and were accompanied by perceptible physiologic alterations. The goals of this study were to verify the influence of trate-anxiety and state-anxiety in psychobiological manifestation of patient in immediate post-operative period. The data were collected using trate and state anxiety inventary, two questionnaires to evaluate clinical conditions of 33 surgical patients. The data were collected in immediate pre-operative and post-operative periods. The results showed a significative correlation between trate and state anxiety in pre-operative period. There was no significative correlation among state anxiety in pre-operative period and clinical conditions in post-operative period. As conclusion, the correlation was no significative among trate anxiety, state anxiety and psychobiological manifestations in post-operative period.

UNITERMS: Anxiety. Postoperative period. Anesthesia recovery period.

\section{INTRODUÇÃO}

No que se refere ao ato anestésico-cirúrgico é possível supor que a antecipação deste evento pelo paciente desencadeará sentimentos e uma avaliação cognitiva, os quais, influenciados pelas diferenças individuais, resultarão em comportamentos de ajustes que têm por finalidade enfrentar o stress e a ansiedade provocados por esse momento.

1 Tese (Doutorado), 1998, EEUSP.

- Professor Doutor do Departamento de Enfermagem Médico-Cirúrgica.

* Professor Doutor do Departamento de enfermagem Médico-Cirúrgico e Professor da Universidade Federal de Curitiba. 
Essas avaliações cognitivas serão mais efetivas quanto mais dados da realidade o indivíduo tiver. Várias são as possibilidades de obtenção destes dados, desde experiências passadas até a formulação de dúvidas, questionamentos, hipóteses e associações de idéias relativas à cirurgia e à anestesia.

Segundo LAZARUS; FOL̇KMAN (1984) avaliação cognitiva é um processo de categorização de um encontro, com o foco no propósito ou no significado desse encontro. Estes autores definem encontro como a aproximação de dois elementos, sendo que um deles é o sujeito e o outro é de natureza diversa e desencadeadora do estímulo.

Para SPIELBERGER (1979), se um estímulo interno ou externo ao sujeito for interpretado como perigoso ou ameaçador desencadeará uma reação emocional caracterizada como um estado de ansiedade.

Esse autor define estado de ansiedade como reação emocional percebida pela consciência e caracterizada por sentimentos subjetivos de tensão, apreensão nervosismo e preocupação intensificando a atividade do sistema nervoso autônomo. Estas respostas incluem alteração da freqüência cardíaca, do padrão respiratório e da pressão arterial, inquietação, estremecimentos, tremores e aumento de sudorese. Afirma que a maneira como o indivíduo percebe a ameaça é mais importante do que a própria ameaça. Considera as diferenças na percepção e as reações às situações como traço de ansiedade, isto é, comportamentos individuais que permanecem latentes, até que em uma determinada situação são ativados. Estes comportamentos são influenciados por experiências passadas que levam os indivíduos a reagir de determinada forma.

Parece não haver dúvida entre os estudiosos da neuroendocrinologia das emoções que existe uma correlação estreita entre a percepção sensitiva e cognitiva de uma ameaça, o surgimento da ansiedade e do stress e alterações fisiológicas perceptíveis clinicamente. No entanto, estas afirmações não são tão claras e definitivas em situações nas quais a avaliação cognitiva está ou esteve temporariamente abolida por qualquer motivo.

Sendo assim o processo anestésico-cirúrgico representa um exemplo de supressão temporária da consciência que implica na incompetência também temporária, do paciente em avaliar cognitivamente a situação a qual está submetido. SPIELBERGER; GORSUCH; LUSHENE (1979) compararam o traço de ansiedade a uma energia potencial que lhe confere a qualidade de energia potencial latente existente em cada indivíduo e que pode ou não ser liberada em determinadas situações. Também é esperado que pessoas com alto traço de ansiedade apresentem elevação de estado de ansiedade, uma vez que elas tendem a considerar as situações como ameaçadoras. Concebem o mundo como mais perigoso do que as pessoas com baixo traço de ansiedade, reagindo mais intensamente ao estímulo.

Em virtude desta situação após a realização da cirurgia, encontra-se no período pós-operatório imediato, em sala de recuperação anestésica (SRA), o indivíduo numa fase de vulnerabilidade e instabilidades, com a sua capacidade psicológica adaptativa alterada, uma vez que o processo de questionamento e busca de dados, que dão suporte à avaliação cognitiva, sofre uma ruptura quando a pessoa é submetida a um procedimento anestésico. Neste caso, os medos e as incertezas tendem a aumentar em função da impossibilidade de se obter dados desse momento.

Surgirão em conseqüência desta experiência, alterações que neste momento, podem ser percebidas por meio de observações clínicas e medidas de parâmetros vitais que vão refletir as condições do paciente, no período de recuperação anestésica.

A complexidade existente na resposta dada pelo homem ao procedimento anestésico-cirúrgico evidencia que os resultados obtidos com a verificação dos parâmetros clínicos precisam ser interpretados como um reflexo do homem operado, isto é, não é só a dor que provoca taquicardia ou um aumento da pressão arterial, assim como este, outros sinais também são decorrentes e potencializados pelos fatores ansiogênicos oriundos do procedimento anestésico-cirúrgico.

$O$ resultado desta avaliação não pode ser interpretado puramente como uma manifestação biológica decorrente do processo anestésico-cirúrgico, mas como uma resposta psicossomática de vivência deste evento.

Mesmo considerando as manifestações orgânicas do homem como respostas a estímulos na sua estrutura biológica e psíquica simultaneamente, na fase pósoperatória imediata essa consideração ainda não encontra espaço para identificação, uma vez que o universo subjetivo do homem e suas manifestações no período transoperatório correspondem a uma situação ainda inacessível dado as características dos instrumentos que se dispõe, até o momento, para avaliação de uma pessoa inconsciente ou semiconsciente.

Em outras palavras não se tem meios para precisar que tipo de manifestação emocional é possível ocorrer durante um processo anestésico, ou até se ela está mesmo presente ou não e portanto, não se tem base para afirmar ou tão pouco negar que elas estejam interferindo nos parâmetros orgânicos da fase pósoperatória imediata.

A partir de então, a questão em torno do modo como a ansiedade se manifesta clinicamente, nesta situação específica, ou seja, como é a expressão somática do stress quando a subjetividade está embotada pelas drogas anestésicas. Na busca de esclarecimentos desta questão, este estudo tem por objetivos: 
Verificar a correlação entre o traço de ansiedade e o estado de ansiedade no período pré-operatório.

Verificar a correlação entre o estado de ansiedade, no período pré-operatório, e a sua manifestação na resposta do paciente no período pós-operatório imediato.

\section{Local de Estudo}

Este estudo foi realizado em um hospital geral governamental. Para o atendimento aos pacientes cirúrgicos, o hospital possui dois Centros Cirúrgicos: um destinado às cirurgias ambulatoriais e um outro, de maior porte, com 34 salas de operações, destinado às diversas especialidades cirúrgicas, atendendo aos pacientes, tanto nas emergências como também nas cirurgias de urgências e eletivas. Como unidade de apoio ao centro cirúrgio existe a Sala de Recuperação Anestésica (SRA).

Nessa sala são recebidos, nas 24 horas de funcionamento, os pacientes submetidos à intervenção anestésico-cirúrgica, provenientes de cirurgias eletivas, de urgências ou emergências. Os pacientes, permanecem em média de 3 a 4 horas nessa sala.

\section{Amostra de estudo}

A amostra estudada constou de 33 pacientes internados nas unidades de Clínica Cirúrgica do Instituto Central para a realização da intervenção anestésico-cirúrgica.

Os critérios para seleção da amostra foram os pacientes: alfabetizados; faixa etária de 20 a 65 anos; pertencentes ao programa de cirurgias eletivas; submetidos à intervenção cirúrgica ginecológicas $\mathrm{e}$ gerais de pequeno e médio porte, segundo FAINTUCH; FAINTUCH (1978); encaminhados à SRA, após o ato anestésico-cirúrgico; sem distúrbios orgânicos, fisiológicos, bioquímicos ou psiquiátricos, estado físico 1, de acordo com o American Society of Anesthesiology ASA 1 (FAINTUCH; FAINTUCH, 1978; HOFFER, 1997) ou com anormalidades fisiológicas, bioquímicas de pequena intensidade, como diabetes mellitus ou hipertensão arterial leve e compensada, estado físico 2, de acordo com American Society of Anesthesiology ASA 2 (FAINTUCH; FAINTUCH, 1978; HOFFER, 1997); que tivessem recebido como medicação préanestésica o benzodiazepínico, midazolan.

\section{Instrumentos:}

\section{Formulário 1}

Parte I: composto por dados gerais de identificação como idade, hospitalização e cirurgia anteriores e cirurgia a ser realizada, pois pressupunha-se que estes dados influenciariam na ansiedade do paciente.

Parte II: contem parâmetros clínicos considerados por diversos autores como constitutivos da avaliação de pacientes cirúrgicos (ALDRETE; KROULIK, 1970;
POSSO, 1975; CASTAÑOS, 1982; SCHINEIDER, 1982; GRIFFIN, 1989), e por estarem associados à atividade nervosa central autonômica e endócrina desencadeadas pela ansiedade (LACHMAN, 1974; DeLUCIA, PLANETA, 1991; McGAFFIGAN; CHRISTOPH, 1994). São eles: freqüência de pulso, pressão arterial, freqüência respiratória, temperatura, reflexos protetores (tosse, deglutição), expansibilidade torácica, atividade muscular, sensibilidade cutânea, dor, coloração, nível de consciência, compor-tamento, sinais vegetativos (vômito, náusea, sialorréia, sudorese) e glicemia de jejum.

\section{Inventários 1 e 2:}

Foram utilizados e aplicados pela autora os inventários propostos por SPIELBERGER; GORSUCH; LUSHENE (1979) yalidados e conhecidos como inventário de Ansiedade Traço - Estado (IDATE). Esses inventários são escalas compostas por duas partes distintas para medir dois conceitos subjetivos: ansiedadetraço e ansiedade-estado. $O$ conceito de ansiedade-traço é definido como a propensão à ansiedade, isto é, a tendência do indivíduo perceber as situações como ameaçadoras, ou seja, como ele geralmente se sente. A ansiedade-estado, segundo ANDREATINI; LEITE (1994), seria transitória, caracterizada por sentimentos desagradáveis conscientemente percebidos, modificandose com o tempo e sendo influenciada por fatores externos. Essas escalas constam de 20 afirmações, em que o sujeito indica a intensidade naquele momento (estado) ou a freqüência com que ocorre (traço), através de uma escala de quatro pontos ( 1 a 4 ). $O$ escore de cada escala varia de 20 a 80 , sendo que os escores mais altos indicam maior nível de ansiedade (SPILBERGER, GORSUCH; LUSHENE, 1979).

Em alguns itens deste inventário, as pontuações devem ser invertidas, já que o conteúdo das afirmações nele contidas é oposto das afirmações dos demais itens. São eles: $1,6,7,10,13,16$ e 19, na parte referente ao estado, e $1,2,5,8,10,11,15,16,19$ e 20 na parte referente ao traço.

\section{Formulário 2:}

Parte I: composto por dados relativos ao tipo de anestesia, tipo de cirurgia realizada, droga pré-anestésica utilizada e tempo de jejum, pois considerava-se que estas informações influenciariam nas comparações e correlações necessárias para este estudo.

Parte II : contém os mesmos parâmetros de avaliação investigados na fase pré-operatória, diferindo na forma de classificar o pulso, pressão arterial, freqüência respiratória, temperatura e glicemia, ou seja, esses sinais vitais e a glicemia receberam a classificação normal (0), alterado (1) e muito alterado (2), de acordo com a variação existente entre os valores obtidos nos períodos pré-operatório e o pós - operatório imediato. 


\section{Procedimento de coleta de dados:}

Foram realizadas entrevistas com os paciente cirúrgicos que preenchiam os critérios de seleção da amostra, informando-os sobre a finalidade da pesquisa, da participação voluntária e da garantia do anonimato, avaliados os parâmetros clínicos, com exceção do valor da glicemia, que foi obtido pela consulta no prontuário, $e$ aplicado os questionários. Estes dados foram coletados em duas fases: 1 a fase: Realizada na enfermaria, no dia anterior a cirúrgia.Os pacientes eram questionados quanto aos dados de identificação e avaliados quanto aos parâmetros clínicos ( frequêencia de pulso e respiratória, pressão arterial e temperatura ). Estes foram considerados como normais, embora correspondessem ao valor basal do paciente, uma vez que se pretendia, ao final da pesquisa, acompanhar a variação ocorrida entre o período pré e pós-operatório.

Os outros parâmetros usados para avaliação clínica no período pré-operatório foram pontuados, com exceção da dor, com base nas variações propostas pelos autores (ALDRETE; KROULIK, 1970; POSSO, 1975; CASTAÑOS, 1982; SCHINEIDER, 1982; GRIFFIN, 1989) e a partir daí criou-se três possibilidades de escores: normal (0), alterado (1) e muito alterado (2), Quanto à avaliação do parâmetro referente à dor, foi baseada na escala proposta por Downie e colaboradores Essa escala é composta por 11 números entre zero a $\operatorname{dez}(0$ a 10$)$. $O$ zero $(0)$ representa um extremo de dor (ausência) e o dez (10) representa o outro extremo (muita dor). JENSEN; KAROLY; BRAUER (1986). A pontuação dada pelo paciente nessa escala foi classificada nesta pesquisa em normal ( 0 ), quando o paciente avaliava sua dor, entre 0 a 3 ; alterada (1), quando o paciente avaliava sua dor entre 4 a 6 e muito alterada (2), quando a pontuação dada era entre 7 a 10. Após a verificação destes parâmetros foram distribuídos 2 questionários auto-aplicáveis validados e conhecidos como Inventário de Ansiedade Traço-Estado (IDATE).

Estes inventários tiveram a finalidade de verificar a ansiedade do paciente cirúrgico no período pré-operatório. Para avaliação dos escores obtidos, optou-se pela categorização utilizada por CHAVES (1994), ou seja, 20 a 40 pontos (baixa ansiedade); 40 a 60 pontos (média ansiedade); 60 a 80 pontos (alta ansiedade).

Ao final de cada avaliação, obteve-se um escore referente ao traço de ansiedade e outro, ao estado de ansiedade.

2a fase: realizada, após a cirurgia, em SRA. A avaliação dos parâmetros clínicos na fase pós-operatória obedeceu aos intervalos estabelecidos pela rotina desta unidade, ou seja, um intervalo de 30 minutos, na primeira hora, e de 1 em 1 hora após este período.

Verificou-se nesta fase os mesmos parâmetros clínicos já avaliados no período pré-operatório, acrescido de tempo de jejum, tipo de anestesia e cirurgia realizadas, medicação pré-anestésica utilizada e classificação quanto ao risco anestésico-cirúrgico.
Estes parâmetros clínicos foram avaliados e classificados em vários momentos do período pósoperatório, isto é, ao chegar (TPOS), após trinta minutos (TPOS1), após uma hora (TPOS 2), após duas horas(TPOS 3) e após três horas de permanência em SRA.

No final de cada momento, obteve-se um escore de respostas do pós-operatório que poderia variar de zero $(0)$ a trinta $(30)$.

Para avaliação dos escores obtidos foi estabelecida a seguinte categorização: 0 a 9 pontos (normal); de 10 a 19 pontos(alterado) e 20 a 30 pontos (muito alterado).

Os inventários 1 e 2 não foram aplicados neste período em virtude da alteração do estado de consciência dos pacientes em fase de recuperação anestésica.

\section{Tratamento Estatístico:}

Para análise dos dados, foram realizados testes não paramétricos com a aplicação da prova "U" de Mann-Whitney, e do coeficiente de correlação por postos de Kendall. A prova "U" de Mann-Whitney (SIEGEL 1975) foi utilizada na comparação dos grupos considerados independentes. As comparações foram realizadas com os escores obtidos na avaliação de traço de ansiedade, estado de ansiedade e os escores recuperação do paciente no pós-operatório, no momento de chegada à sala de recuperação anestésica (TPOS). Os demais escore totais obtidos com a avaliação dos parâmetros clínicos do período pós-operatório (TPOS, TPSO2, TPSO3 e TPSO4) não foram utilizados para análise por não apresentarem diferenças estatísticas entre aqueles obtidos com a avaliação feita na chegada SRA, TPOS e trinta minutos após de permanência nesta sala (TPOS1).

Tanto a prova de Mann-Whitney como o coeficiente de correlação de Kendall foram testados quanto às respectivas significâncias, através da aproximação normal, de modo unicaudal. A probabilidade de ocorrência do erro de primeira espécie foi fixada em $5 \%$ e o valor crítico dos testes $(Z)$ foi 1,64 .

\section{RESULTADOS E DISCUSSÃo}

\section{Caracterização da Amostra:}

Participaram desse estudo 33 pacientes submetidos a cirurgias eletivas, sendo $23(69,7 \%)$ mulheres e $10(30,3 \%)$ homens . A amostra foi constituída em sua maioria por mulheres. Essa distribuição, pode ser decorrente de um dos critérios adotados para a seleção da amostra, pois a opção pelas cirurgias de pequeno e médio porte, nesse período e nesse hospital, levou a uma maior freqüência de pacientes do sexo feminino. 
Com relação idade, os dados apresentados na tabela 1 mostram que amostra é composta por indivíduos adultos.

Segundo a experiência pregressa, encontrou-se uma porcentagem relevante de pacientes $(21,2 \%)$ sem experiência de hospitalização anterior com cirurgia, e outros $78,8 \%$ dos pacientes com alguma referência simbólica relativa à vivência de um evento cirúrgico. Isto pressupõe alguma experiência anterior armazenada em sua memória a qual, de certo modo, trará para a avaliação das situaçõesatuais afetos e enfrentamentos impregnados das situações vividas anteriormente.

Quanto ao risco cirúrgico apresentado pelos pacientes da amostra, a classificação obtida, segundo a American Society Anesthesiology (ASA) foi de 27 pacientes $(81,8 \%)$ classificados em ASA 1 , o que os identifica como estando em bom estado geral, sem moléstias intercorrentes; $6(18,2 \%)$ pacientes pertencentes ao grupo ASA 2 eram hipertensos ou diabéticos compensados. Esta classificação dada pela ASA considera estas moléstias, quando controladas, como intercorrentes, não acrescentado risco ao paciente no ato anestésico-cirúrgico. Ou seja, pela avaliação da ASA, no período pré-operatório, os pacientes dessa amostra foram classificados em sua maioria em ASA 1, o que induz a uma pressuposição de que o pós-operatório do grupo seria sem intercorrências.

Observou-se que a maioria dos pacientes, 24 $(72,7 \%)$, foi submetida à anestesia geral, enquanto que outros $9(27,3 \%)$, à anestesia regional, que, nesse grupo específico, refere-se aos bloqueios subaracnóides (raquianestesia e a peridural). Neste trabalho foram considerados como um só grupo.
Esta proporção pode estar associada ao tipo de cirurgia realizada, sendo que as cirurgias laparoscópicas e a biópsia de nódulos mamários para diagnósticos (19) foram responsáveis pelo maior número de anestesia geral em conjunto com as herniorrafias (5).

No que se refere ao tipo de cirurgia realizada, a amostra, segundo os critérios preestabelecidos, apresentou $23(69,7 \%)$ pacientes submetidos à cirurgia ginecológica e $10(30,3 \%)$ a cirurgias gerais. Com relação às cirurgias ginecológicas, estas corresponderam à biópsia de nódulo mamário (2); laparoscopias diagnósticas (13); histerectomia (5) e ooforectomia (3). As herniorrafias inguinal, incisional e umbilical foram escolhidas dentre as cirurgias gerais.

Quanto ao traço e estado de ansiedade, a amostra apresentou a distribuição de $16(48,4 \%)$ pacientes com escore para baixo traço de ansiedade $(20$ - 40) e $17(51,5 \%)$ com escore para médio traço de ansiedade $(40-60)$. Com relação ao escore para estado de ansiedade, $23(69,7 \%)$ dos pacientes apresentam baixo estado de ansiedade, 7 $(21,2 \%)$, médio estado de ansiedade e $3(9,1 \%)$, alto estado de ansiedade, o que caracteriza a amostra como sendo pouco ansiosa e respondendo com pouca ansiedade ao evento cirúrgico.

Descreve-se a seguir os dados apresentados na tabela 2. Verifica-se que os valores referentes aos escores para traço e estado de ansiedade são estatisticamente significantes e menores nos pacientes de sexo masculino quando comparados aos do sexo feminino ( $\mathrm{z}$ traço. $=1,80 \mathrm{e} \mathrm{z}$ estado. $=1,72$ ). Para a variável resposta no pós-operatório, não houve diferença significante quando comparados os valores entre os pacientes masculinos e femininos $(z=0,80)$. 


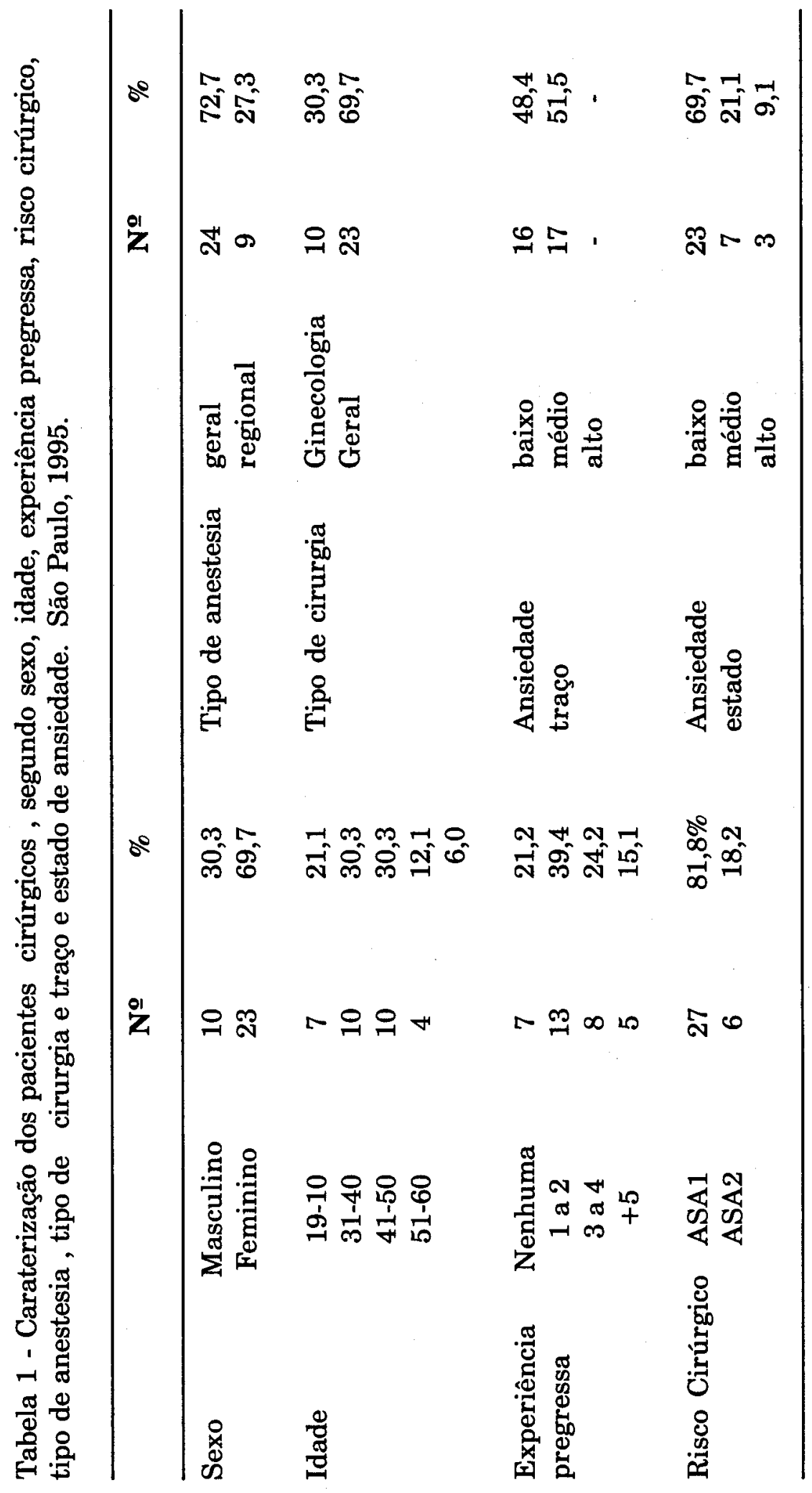




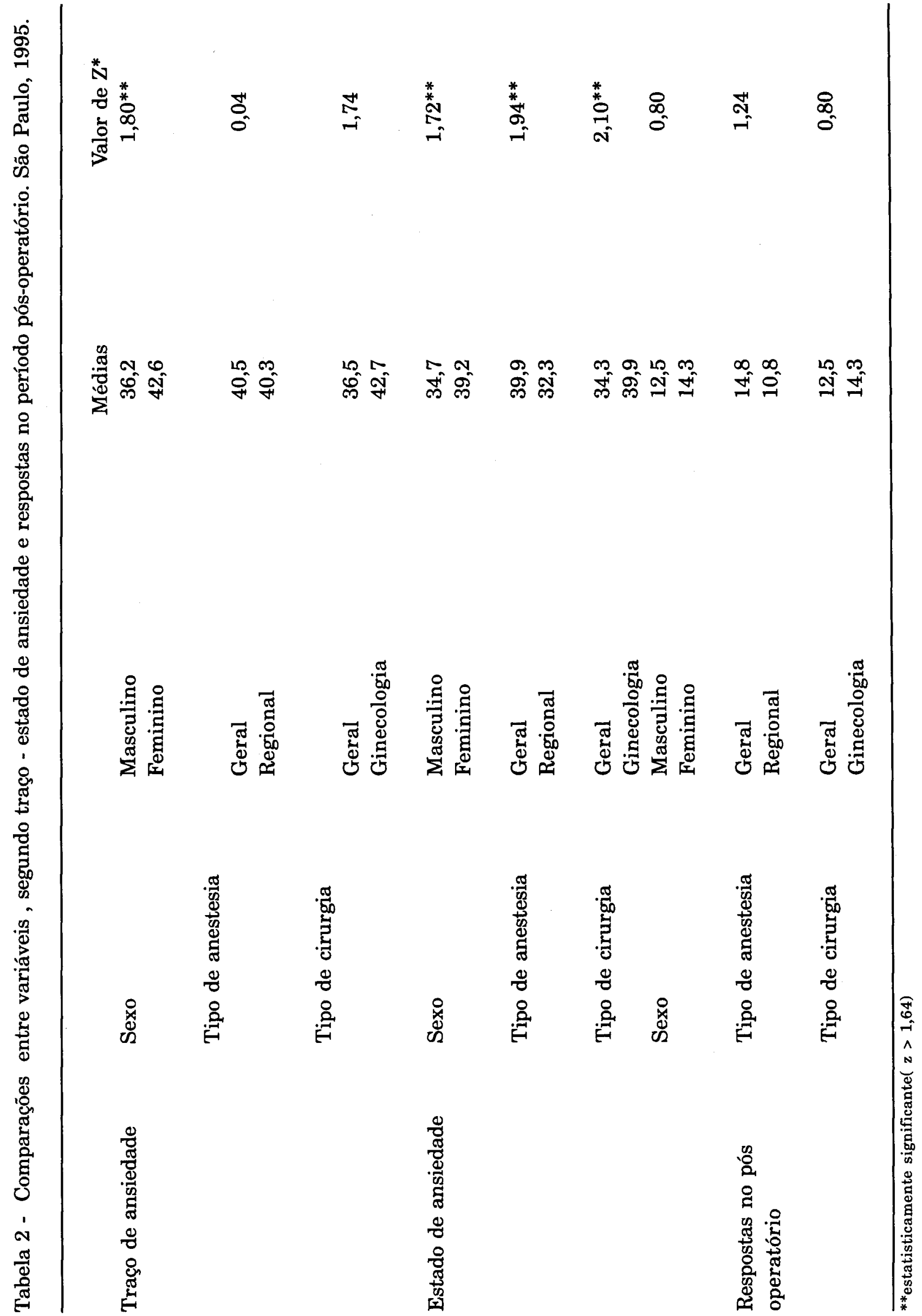


Embora esta diferença não tenha sido estatisticamente significante, ela mostrou-se relativamente correspondente ao traço e ao estado de ansiedade, em ambos os sexos, o que permite concluir que houve correspondência entre os escores obtidos com os inventários auto-aplicáveis e, portanto, subjetivos com os parâmetros observáveis clinicamente.

As médias femininas $(42,6$ e 39,2$)$ foram mais elevadas, tanto para o traço como para o estado de ansiedade respectivamente, mostrando variação relacionada com o traço e estado de ansiedade maior para o sexo feminino do que para o masculino. $(36,2 \mathrm{e}$ $34,7)$ Esta variação demonstra que as mulheres da população estudada eram mais ansiosas que os homens. No entanto, responderam com menor ansiedade que eles ao evento cirúrgico. Isto é, a média de estado de ansiedade masculina diminui 1,5 pontos, em relação à média de traço de ansiedade, enquanto que a média de estado de ansiedade feminina diminui 3,4 pontos em relação à média referente ao traço de ansiedade. Diante disso, é possível supor que as mulheres tenham apresentado mecanismos de enfrentamento do stress e da ansiedade mais eficientes que os homens.

Com relação à categorização feita para avaliar a resposta no pós-operatório, os homens e as mulheres obtiveram valores médios de 12,5 e 14,3 pontos respectivamente, os quais são classificados como alterados (10-19). Esta variação, apesar de importante, não se mostrou estatisticamente significante $(z=0,80)$.

Os valores referentes ao traço de ansiedade não apresentaram diferença significante $(\mathrm{z}=0,04)$ quando comparados ao tipo de anestesia realizada. No que se refere ao estado de ansiedade, houve diferença estatisticamente significante entre os pacientes submetidos à anestesia geral e ao bloqueio anestésico $(z=1,94)$.

Esta diferença entre traço e estado de ansiedade pode estar relacionada com o fato de os pacientes terem conhecimento do tipo de anestesia a que seriam submetidos e elaborado uma fantasia de maior risco associada à anestesia geral. Por outro lado, no que se refere à recuperação global dos pacientes, é possível que as maiores alterações clínicas apresentadas nos pacientes submetidos à anestesia geral, estiveram relacionadas ao stress de origem orgânica, uma vez que, no momento em que foram verificados (TPOS) os pacientes, em sua maioria encontravam-se semiconscientes, independente do tipo de anestesia que receberam.

Ao se comparar os valores referentes ao traço, estado de ansiedade e resposta no pós-operatório, segundo o tipo de cirurgia, observa-se que os indivíduos submetidos a herniorrafias têm escores menores nas três variáveis em questão quando comparados aos indivíduos submetidos a cirurgias ginecológicas.

Os dados evidenciaram que as mulheres apresentaram traço e estado de ansiedade maiores que os homens. É importante retornar a esta informação, uma vez que as cirurgias ginecológicas estiveram relacionadas à população feminina, assim como as herniorrafias, à população masculina. Este dado por si só seria suficiente para justificar o resultado obtido,no entanto, vale acrescentar que a própria natureza da cirurgia é outro fator que pode ter influenciado nos resultados, isto é, as inúmeras conseqüências caso 0 prognóstico de câncer fosse confirmado ou ainda, o resultado da laparoscopia diagnóstica não fosse compatível com as expectativas.

Resultados semelhantes foram encontrados por MARTIN (1996), em estudo feito com pacientes cirúrgicos. Ele chegou à conclusão de que as mulheres participantes do grupo apresentaram alteração maior no estado de ansiedade do que os homens, a qual poderia ser justificada pelo fato de terem sido submetidas a cirurgias que envolveram mudança da auto-imagem.

Da mesma forma, os estudos de NYAMATHI; KASHIWABARA (1988) demonstraram que as mulheres submetidas a cirurgias ginecológicas tiveram aumento de ansiedade quando comparadas a outros pacientes cirúrgicos, mesmo tendo sido excluídas as cirurgias diagnósticas.

\section{CORRELAÇÃo ENTRE AS VARIÁVEIS}

Neste item, serão apresentadas e analisadas as correlações entre traço e estado de ansiedade, no período pré-operatório e a resposta no pós-operatório em SRA.

Segundo SPIELBERGER (1979), é esperado que indivíduos com alto traço de ansiedade respondam com elevação do estado de ansiedade às situações.

$\mathrm{Na}$ amostra estudada, observou-se um comportamento do traço de ansiedade em relação ao estado de ansiedade compatível com esse conceito, pois, houve correlação significativa e positiva entre traço e estado de ansiedade para cada sexo com valores de zmas $=2,10$ e zfem $=1,80$ conforme apontam os dados da tabela 3 . 
Tabela 3 - Medidas de correlação entre as variáveis estado de ansiedade e traço de ansiedade, hospitalização anterior , idade, parâmetros clínicos do período pré-operatório e a resposta no período pós-operatório. São Paulo, 1995.

\section{Coeficiente \\ de correlação \\ de Kendall}

Valor de $\mathrm{Z}^{*}$

\begin{tabular}{|c|c|c|c|c|}
\hline \multirow{14}{*}{$\begin{array}{l}\text { Estado de } \\
\text { ansiedade }\end{array}$} & \multirow[t]{2}{*}{ traço de ansiedade } & Masculino & 0,49 & $2,10^{* *}$ \\
\hline & & Feminimo & 0,28 & \\
\hline & \multirow{2}{*}{$\begin{array}{l}\text { hospitalização } \\
\text { anterior }\end{array}$} & masculino & $-0,09$ & 0,40 \\
\hline & & feminimo & 0,11 & 0,72 \\
\hline & \multirow[t]{3}{*}{ idade } & masculino & 0,04 & 0,40 \\
\hline & & feminino & 0,06 & 0,72 \\
\hline & & & & 0,38 \\
\hline & \multirow{6}{*}{$\begin{array}{l}\text { parâmetros } \\
\text { clínicos (pré- } \\
\text { operatórios) }\end{array}$} & & $P=0,09$ & 0,75 \\
\hline & & & $\mathrm{PAS}=0,00$ & 0,02 \\
\hline & & & $\mathrm{PAD}=0,04$ & 0,33 \\
\hline & & & $R=0,11$ & 0,90 \\
\hline & & & $\mathrm{T}=0,12$ & 1,00 \\
\hline & & & $\mathrm{GL}=0,03$ & 0,27 \\
\hline & \multicolumn{2}{|l|}{$\begin{array}{l}\text { Resposta no pós- } \\
\text { operatório } \\
\text { (TPOS) }\end{array}$} & 0,13 & 1,09 \\
\hline
\end{tabular}

Estado de

\begin{abstract}
traço de ansiedade
\end{abstract}
hospitalização

terior

** estatisticamente significante $(z>1,64)$

Estes resultados são opostos aos encontrados por SPIELBERGER et al (1973) que, avaliando a reação emocional de homens submetidos a cirurgias, encontraram escores altos para estado de ansiedade em pacientes com alto e baixo traço de ansiedade. Concluíram, com isso, que $o$ ato cirúrgico elevou o estado de ansiedade da população do estudo a níveis altos e semelhantes, independente do traço de ansiedade identificado.

É importante salientar que, mesmo apresentando uma correlação positiva entre o traço e o estado de ansiedade, 19 indivíduos da amostra apresentaram escore para o estado de ansiedade menor do que seu traço de ansiedade no período pré-operatório.

Tanto para a afirmação relativa à proporcionalidade entre traço e estado, quanto para a afirmação que o estado se eleva em relação ao traço, no período pré-operatório, o presente estudo apresentou dados que se opõem aos encontrados por SPIELBERGER et al (1973). Esta diferença pode estar relacionada com o tipo de população, de cirurgia, relação estabelecida entre a equipe de saúde e os pacientes e o conteúdo de informações recebidas pelas diferentes populações.
Ainda sobre a variação individual, SPIELBERGER (1979) afirma que a correspondência entre traço e estado de ansiedade depende de como a situação específica é percebida pelo indivíduo. A inversão da relação traço e estado de ansiedade, na população do presente estudo, talvez possa ser justificada por estilos de coping eficazes, que casualmente essa amostra apresentou, ou ainda, por algumas outras razões associadas à dificuldade na compreensão dos itens que compõem os questionários, comprometendo a fidedignidade da resposta, ou ainda a alteração da capacidade de abstração em decorrência de o paciente estar vivenciando uma situação que não lhe é comum.

WAITZBERG; HOJAIJ (1985), em estudo preliminar sobre a influência da ansiedade no período pós-operatório, também apontam como uma das dificuldades a dúvida da veracidade nas respostas dadas pelos pacientes aos inventários auto-aplicáveis, pois consideram que esses poderiam, inconscientemente, não fornecer a informação precisa, com receio de punição, ou porque estariam dando sinais de fraqueza perante a internação e a cirurgia. 
SPIELBERGER et al (1973), ao avaliarem a reação emocional de pacientes submetidos à cirurgia, chamaram a atenção para "ansiedade inconsciente" não detectada por muitos testes, a qual apesar de estar presente, não é percebida, levando a uma falsa avaliação.

Esses autores vão além dos problemas da compreensão e conseqüências da avaliação na hospitalização. Pontuam que o próprio estado emocional atual do paciente pode interferir nos resultados. Citam que o momento em que o inventário é aplicado pode influenciar, pois a ansiedade medida relaciona-se também com as preocupações familiares, aos aspectos financeiros e não só com o procedimento anestésicocirúrgico em si.

Nesse estudo, além dos fatores citados, as informações recebidas pelo paciente dos vários membros da equipe médica e de enfermagem podem ter contribuído para a inversão entre traço e estado de ansiedade.

Apesar de não existir uma forma estruturada de orientação pré-operatória na Instituição onde se efetuou a coleta de dados, o paciente recebeu orientações relacionadas com 0 ato anestésico-cirúrgico das equipes, além da troca de informação existente entre os companheiros da clínica, o que pode ter influenciado nos resultados obtidos.

A presença da pesquisadora, por mais cuidadosa que tenha procurado ser, no sentido de não interferir nos resultados, também pode ter contribuído com o fato, pois ao abordar o paciente no período pré-operatório, lhe assegurou a presença após a cirurgia. Acredita-se que numa situação delicada, poder encontrar alguém com quem se tivesse mantido um contato anterior possa contribuir para que o estado de ansiedade no período pré-operatório não se altere acentuadamente. Neste sentido, JOUCLAS; SALZANO (1981); BIANCHI (1990); SILVA (1987); MARTIN (1996) relatam a importância da interação interpessoal para a diminuição da ansiedade do paciente, que contribui para a evolução mais tranquiila no período pós-operatório.

Por outro lado CALDWELL, (1991), em estudos realizados com pacientes cirúrgicos, revelou que alguns não se interessavam em receber mais informações além daquelas já obtidas, pois referiam um aumento de stress com o excesso de informações no período pré-operatório. Assim como considera COSTA (1997), as informações e esclarecimentos de dúvidas, na fase pré-operatória devem ocorrer de uma forma que o paciente possa interagir e participar, cabendo ao enfermeiro observar, ouvir e descobrir suas reais necessidades, utilizando-se da comunicação como um procedimento terapêutico.

Nenhuma das constatações feitas e corroboradas deve ser analisada unilateralmente. SPIELBERGER (1979) salienta a importância dos processos mentais compostos por percepção, pensamento, memória e julgamento, na avaliação subjetiva de uma situação; que poderá ou não levar à ansiedade. No que se refere ao presente estudo, a percepção, a memória e o julgamento relacionados com a cirurgia, anestesia e hospitalização podem ter contribuído para gerar maiores ou menores oscilações no estado de ansiedade, assim como a própria característica de ansiedade de amostra deste estudo.

Além disso, as experiências anteriores do indivíduo podem alterar o seu estado de ansiedade atual, isto é, o paciente cirúrgico, de um modo geral, pode ter seu estado emocional, no período pré-operatório, modificado pelas lembranças das hospitalizações e cirurgias anteriores. Segundo DALLY; HARRINGTON (1978), quando se evoca um fato, tem-se a memória ativada, desencadeando modificações no estado emocional, que dependerão dos afetos contidos nas lembranças resgatadas. $\mathrm{Na}$ população do presente estudo, como já foi apresentado, $78,8 \%$ dos pacientes tiveram experiências cirúrgicas anteriores o que, embora não tenha apresentado correlação estatística significante ( zmas $=-0,09$ e zfem $=$ $0,11)$, nos permite afirmar que suas respostas à situação atual estiveram vinculadas a estas vivências anteriores, diminuindo possivelmente a reação emocional ansiosa diante do desconhecido.

É importante salientar que a significância estatística se dá a partir da oscilação numérica. No entanto, neste caso, o que ocorre é uma incompatibilidade entre o que é qualificado como significante para a estatística e o que é significante para a análise da ansiedade, isto é, o fato de haver pouca variação entre as médias de escore de traço e estado de ansiedade constitui informação relevante, uma vez que se espera, via de regra, diante de qualquer evento ameaçador ou desafiador, um aumento da segundo em relação ao primeiro. Algo relevante ocorreu na população em questão, capaz de opor-se à tendência apontada universalmente na literatura (SPIELBERGER et al, 1973; SPIELBERGER; GORSUCH; LUSHENE, 1973 ; SPIELBERGER, 1979).

No que diz respeito à associação do estado de ansiedade e a idade, neste estudo, não se obteve correlação significante entre estas duas variáveis, ou seja, o estado de ansiedade mostrou-se, na sua maioria, em níveis baixos independente da idade e do sexo dos sujeitos investigados.

Conforme já apresentado anteriormente sempre que uma situação for considerada ameaçadora, independente de ser real ou imaginária, ocorrerá uma reação emocional definida como estado de ansiedade acompanhado de alterações fisiológicas (SPIELBERGER, 1979)

É esperado, portanto que a aproximação do ato anestésico-cirúrgico, por ser considerado um evento ameaçador, desencadeie essa reação emocional com suas correspondentes alterações orgânicas, isto é, um aumento da atividade do sistema nervoso simpático com alteração da freqüência de pulso, respiração, pressão arterial, temperatura e glicemia, mudanças estas 
também desencadeadas e reforçadas pelo eixo hipotálamo-hipófise-adrenocortical.

Apesar da ansiedade desencadear toda a reação simpática, neste estudo, não houve significância entre as medidas de freqüência de pulso, de pressão arterial sistólica, de pressão arterial diastólica, de freqüência respiratória , da temperatura e da glicemia no préoperatório. Não foi observada a correlação entre o estado de ansiedade e os sinais compatíveis com o aumento da atividade do sistema nervoso simpático, aqui consideradas com base no referencial teórico utilizado, como manifestações clínicas da ansiedade conforme mostram os dados da tabela 3.

Este fato pode ser decorrente da característica da amostra em possuir médio traço de ansiedade (4060) e exibir, frente a uma situação, um baixo estado de ansiedade (20-40), talvez, por não considerá-la ameaçadora ou avaliá-la como benigna.

Estes dados indicam que não houve correspondência entre a avaliação do estado de ansiedade obtido de forma subjetiva, isto é, a partir de um questionário auto-aplicado com os parâmetros clínicos obtidos de maneira objetiva.

Tal resultado sugere que o questionário não foi fiel ao seu objetivo de dimensionar a ansiedade do paciente ou esta ansiedade não foi transformada de maneira equivalente em sinais orgânicos.

Também não foi encontrada diferença estatisticamente significante entre o estado de ansiedade no período pré-operatório e o escore resposta do paciente no período pós-operatório para ambos os sexos como se observa na tabela 3.

Com o objetivo de dar destaque aos dados mais imediatamente relacionados com a ansiedade, foram feitos testes de correlação entre o estado de ansiedade do período pré-operatório e os valores de pulso, pressão arterial sistólica e diastólica, freqüência respiratória e glicemia no período pós-operatório. Obteve-se como resultado a ausência da correlação estatisticamente significante $(z<1,64)$. Este resultado pode apontar para o fato de que há realmente uma imprecisão, entre o inventário utilizado e a sua correspondência orgânica, comojá foi apontado anteriormente. Outra possibilidade está relacionada com a possível interferência da droga anestésica e pré-anestésica, utilizada, justamente no limite dos dois momentos preestabelecidos para avaliação, neste estudo, e que podem ter interferido nos processos emocionais e orgânicos.

Segundo GUYTON(1986), a droga pré-anestésica tem sua ação diretamente ligada ao sistema límbico e cumpre seus princípios, através do relaxamento mental e emocional, redução dos estímulos sensoriais, redução de taxa metabólica, redução da excitabilidade reflexa, anti-stress, anti-secretória e anti-reflexos das vias respiratórias, durante o procedimento anestésicocirúrgico e período pós-operatório imediato.
O avanço no conhecimento dos substratos neurais da ansiedade possibilitou que novas e potentes drogas fossem testadas e utilizadas como ansiolíticos, o que muito tem auxiliado os pacientes, uma vez que protegem os principais sistemas neurais envolvidos com os comportamentos de luta e fuga. Em experiências laboratoriais desenvolvidas, os benzodiazepínicos começaram a ser utilizados com grande vantagem pela segurança e eficácia no tratamento da ansiedade. $O$ seu efeito ansiolítico ficou demonstrado pela capacidade dos receptores no cérebro em reconhecê-lo, mesmo sendo composto por moléculas artificiais obtidas por síntese química. (GRAEFF, 1983).

Neste estudo, os pacientes receberam como droga pré-anestésica o midazolam, um benzodiazepínico hidrossolúvel, hipnótico sedativo com propriedades ansiolíticas e amnésticas e, como as outras drogas do mesmo grupo, não causam amnésia retrógrada. TONELLI et al (1991) constataram que, 35 pacientes utilizando o midazolan como pré-anestésico tiveram lembranças anteriores preservadas. Por isso, é possível supor que os mesmos preservem alguma emoção que pode ou não ser a ansiedade. A utilização deste benzodiazepínico, como medicação pré-anestésica prescrita aos pacientes deste estudo, pode justificar a não correlação encontrada entre o estado de ansiedade e as manifestações da ansiedade obtida com a avaliação dos parâmetros clínicos, no período pós-operatório, uma vez que a estimulação adrenocortical causada no período pré-operatório ficou reduzida pela droga pré-anestésica.

PARRIS et al (1988) ao estudar a relação entre a ansiedade pré-operatória e a recuperação pósoperatória, em pacientes submetidos à cirurgia ambulatorial, verificaram que só para aqueles pacientes com elevado nível de ansiedade, antes da cirurgia, existe a propensão de aumento de riscos e complicações pós-operatórias.

A estabilidade dos sinais vitais relacionados com as manifestações clínicas da ansiedade pode estar associada à propriedade do benzodiazepínico utilizado. Segundo GRAEFF (1983) De LUCIA; PLANETA (1984), o midazolan, diminui acentuadamente a hipertensão, a taquicardia e a hiperventilação, produzidas por estímulos desagradáveis, o que justificaria a ausência de correlação significativa entre o escore de resposta no pós-operatório e o estado de ansiedade no pré-operatório.

Com relação a esta característica da droga préanestésica, estudos realizados por GUTIERREZ et al (1995) mostraram que grupos de pacientes submetidos à cirurgia de catarata, sob anestesia regional, recebendo como medicação pré-anestésica, o midazolam ou o diazepam, ou ainda, o alfentanil, tiveram a freqüência cardíaca, respiratória, pressão arterial e saturação arterial de hemoglobina sem alterações significantes, quando correlacionadas ao tipo de droga pré-anestésica utilizada. 
Este estudo vem reforçar a idéia de que estas drogas atuam indistintamente sobre os parâmetros vitais, no sentido de minimizar influências límbicas nas suas variações.

No período pós-operatório, além das medidas de pressão arterial, freqüência de pulso, freqüência respiratória, temperatura e glicemia, outros parâmetros foram avaliados, entre eles, a expansibilidade torácica, a atividade muscular, a sensibilidade cutânea, a dor, a coloração, o nível de consciência, o comportamento, os sinais vegetativos. Estes parâmetros estiveram contidos nos limites de 10 e 19 pontos, numa categorização que variou de 0 a 30 pontos para todos os sujeitos da amostra estudada, no período correspondente à chegada à SRA (TPOS) e após trinta minutos (TPOS1).

Vale salientar que ao chegar à SRA, alguns destes parâmetros avaliados como dor, sensibilidade cutânea e atividade muscular estavam intimamente ligados ao nível de consciência do paciente o que contribuiu para aumentar a dificuldade da avaliação. Corroborando CASTANOS (1982), a consciência sofre uma oscilação, neste período pós-operatório, decorrente da natureza do anestésicos utilizados. Com isto existiu, principalmente na chegada à SRA, dificuldade em avaliar os itens ligados à consciência.

Com relação à avaliação da dor pelo paciente, ressalta-se que é extremamente complexa e influenciada pela personalidade, em seu limiar e tolerância além do que está vinculada ao nível de consciência, o que exige uma avaliação cognitiva, que neste momento estava, pelo menos, parcialmente embotada pelas drogas.

No que se refere à ansiedade, SOFAER (1994) ressalta que alguns pacientes tendem a elevar a percepção dolorosa quando possuem uma alta ansiedade. Os indivíduos desta amostra, em sua maioria, pertencem a uma faixa que varia entre baixo e médio traço-estado de ansiedade, portanto não integrantes do grupo que teria elevação de percepção à dor.

Para OBERLE et al ( 1990) e LIU; BARRY; WEINMAN (1994), um aumento de ansiedade em pacientes submetidos à anestesia geral está correlacionado à dor, no período pós-operatório, sendo que a relação existente entre estado de ansiedade pósoperatório é com o medo da dor e não com o estado de ansiedade anterior à cirurgia. A afirmação desses autores, no entanto, não afastou completamente a importância do estado de ansiedade no pré-operatório. Consideram que ela, está vinculada a um traço de ansiedade. $\mathrm{O}$ medo da dor no pós-operatório seria apenas mais uma manifestação do traço desencadeado por um evento circunstancial.

A dificuldade de se investigar a presença de dor esteve ligada ao período em que se deu essa investigação, isto é, no período pós-operatório imediato, pois, ainda nesta fase, os pacientes estariam sob o efeito analgésico das drogas, como também tinham sua percepção cognitiva e sensitiva alterada pelas mesmas. Vale lembrar que, à semelhança da dor, os demais parâmetros clínicos investigados não se apresentaram muito alterados (com pontuação acima de 20), apesar da avaliação destes não depender do nível de consciência.

Se é possível associar, de alguma forma, a vivência da dor com as oscilações de parâmetros vitais, então a avaliação clínica obtida neste estudo permite inferir que a amostra não vivenciou dor muito profunda no momento em que os dados foram colhidos.

Frente aos resultados obtidos torna-se necessário que outros estudos sejam desenvolvidos no sentido de aprofundar a análise sobre a influência da ansiedade nos pacientes em pós-operatório imediato, buscando a investigação de outras variáveis diretamente relacionadas à esta relação

Esses novos estudos, sem dúvida, abrirão caminhos para pesquisa na área de enfermagem perioperatória e conseqüentemente trarão uma melhoria da assistência de enfermagem, contribuindo para se ter uma visão, sob outros ângulos, da ansiedade no paciente cirúrgico.

\section{CONCLUSÕES}

Existiu uma correlação significativa e positiva entre o traço e o estado de ansiedade no período préoperatório, ou seja, houve uma variação do estado de ansiedade nos pacientes compatível com traço de ansiedade frente ao estímulo da cirurgia e da anestesia;

$O$ estado de ansiedade no período pré-operatório não interferiu na resposta do paciente no período pósoperatório imediato, não apresentando uma correlação significativa entre estas duas variáveis correlacionadas.

\section{REFERÊNCIAS BIBLIOGRÁFICAS}

ALDRETE,J.A.; KROULIK, D. A postanesthetic recovery score. Curr. Rev. Anesth., v. 49, n. 6, p. $924-33,1970$

ANDREATINI,R.; LEITE,J. R. IDATE- traço: adaptação para avaliação da ansiedade durante sete dias, J.Bras. Psiq., v. 43 , n. 5 , p. $259-65,1994$

BIANCHI, E. R. F. Estresse em enfermagem: análise da atuação do enfermeiro de centro cirúrgico. São Paulo, 1990. 117p. Tese (Doutorado)-Escola de Enfermagem, Universidade de São Paulo.

CALDWELL, L.M. Surgical out patients concerns-what every perioperative nurse should know. AORN J., v. 53, n. 3 , p. $761-7,1991$. 
CASTAÑOS, C.C. Indices de recuperação. Rev. Bras. Anestesiol., v. 32, n. 6, p. 441-2, 1982.

CHAVES, E. C. Stress e trabalho do enfermeiro: a influência de características individuais no ajustamento e tolerância ao turno noturno. São Paulo, 1994. 138 p. Tese (Doutorado) - Instituto de Psicologia, Universidade de São Paulo.

COSTA, A. L. S. Análise do stress nas situações de vida diária e do pré-operatório imediato de pacientes cirúrgicos urológicos._São Paulo, 1997.154p. tese ( Mestrado)- Escola de Enfermagem, Universidade de São Paulo.

DALly, P. HaRRINGtoN, H Psicologia e psiquiatria na enfermagem. São Paulo, EPU, 1978. cap.1, p. 3-81: Psicologia.

De LUCIA,R.; PLANETA,C. S. Ansiolíticos In: VALLE, L.B. de $S$. et al. Farmacologia integrada: fundamentos farmacológicos da terapeutica. São Paulo, Atheneu, 1991. cap. 7 , p. 87-91.

FAINTUCH, J.; FAINTUCH, J. J. Observação clínica préoperatória. In: FAINTUCH, J. ; MACHADO, M. C. C. ; RAIA, A. A. Manual de pré e pós -operatório. São Paulo, Manole, 1978. cap. 1, p. 3-13.

GUTIERREZ, R. B. et al Niveis de cortisol em cirurgia de catarata sob anestesia regional. Rev. Bras. Anestesiol., v. 45, p. 274, 1995. Supl. 19.

GRAEFF, F. Psicobiologia da ansiedade. J. Bras. Psiquiatr., v. 32, n. 6, p. $345-50,1983$.

GRIFFIN, D.N. A tool to develop standarts of quality in PACUS. J. Post. Anesth. Nurs., v. 4, n. 2, p. 99-102, 1989.

GUYTON, A C Tratado de fisica médica . 6ed . Rio de Janeiro, Guanabara, 1986. cap. 56, p.606-14: Funções cerebrais do comportamento: sistema límbico, papel do hipotálamo e o controle das funções orgânicas vegetativas.

HOFFER, J.L. Anestesia. In: MEEKER, M.H.; ROTH ROCH, J.C. Alexander cuidados de enfermagem ao paciente cirúrgico. 10 ed. Rio de Janeiro, Guanabara Koogan, 1997, cap. 6 , p. 134-68, 1997.

JENSEN,M. P.; KAROLY,P.; BRAUER, S. The measurement of clinical pain intensity: a comparison of six methods. Pain, v. 27, p. 117-26, 1986.

JOUCLAS, V. M. G. ; SALZANO, S. D. T. Planejamento de uma ficha pré-operatória de enfermagem. Rev. Esc. Enf. USP, v. 15, n. 1 , p. 5-16, 1981.

LAZARUS, R.S.; FOLKMAN,S. Stress appraisal and coping. New York, Springer, Publishing Company, 1984. cap. 2, p. 22-54: Cognitive appraisal process..

LACHAMAN, S. J. Disturbíos psicossomáticos: uma interpretação behaviorista. Rio de Janeiro, Livros Técnicos e Científicos, 1974.
LIU, R; BARRY, J.E.; WEINMAN, J. Effects of background stress and anxiety on postoperative recovery. Anaesthesia, v. 49 , n. 5, p. $382-86,1994$.

MARTIN, D. Pre-operative visits to reduce patient anxiety: a study. Nurs. Stand., v. 10, n. 23, p. 33-8, 1996.

McGAFFIGAN, P. A.; CHRISTOPH, S.B. Asssement and monitoring of the post anesthesia patient. In: DRAIN, C. B. The post anesthesia care unit: a critical care approach to post anesthesia nursing. Philadelphia, W. B. Saunders company, 1994. Chap 19, p. 261-88.

NYAMATHI, A ; KASHIWABARA, A . Preoperative anxiety its affect on cognitive thinking. AORN J., v. 47, n. 1, p. 164-70, 1988.

ORBELE, K. et al. Environment, anxiety and postoperative pain. West. J. Nurs. Res.,v. 2, n. 6, p. 745-57, 1990.

POSSO,M.B.S. Avaliação das condições dos pacientes na sala de recuperação pós-anestésica . Rev. Esc. Enf. USP, v. 9, n. 3, p. 9-23, 1975.

PARRIS, W. C. V. et al. Anxiety and postoperative recovery in ambulatory surgery patients. Anesth. Prog., v. 35, p. 61-4, 1988.

SCHINEIDER,M. Meeting the criteria for discharge. Curr. Rev. Recov. Room Nurses., v. 4, n. 6, p. 41-8, 1982.

SIEGEL, S. Estatística não paramétrica. São Paulo. Mc Grawtill, 1975 .

SILVA,A. A visita pré-operatória de enfermagem realizada pela enfermeira do centro cirúrgico. Rev. Esc. Enf. USP, v. 21 , n. 2, p. 145-60, 1987.

SOFAER, B. Dor manual prático. 2.ed. Rio de Janeiro, Revinter, 1994. (Manual médico de bolso, 9). cap. 3, p. 31-44: A individualidade do paciente.

SPIELBERGER, C. Tensão e ansiedade, São Paulo ,Harper \& Row do Brasil, 1979.

SPIELBERGER, D. et al. Emotional reactions to surgery. J. Consult. Clin. Psychol., v. 40, n. 1, p. 33-8, 1973.

SPIELBERGER, C. ; GORSUCH, R. L. ; LUSHENE, R. E. Inventário de ansiedade traço-estado. Trad. por BIAGGIO, A.M.B.; NATALICIO, L. Rio de Janeiro, CEPA, 1979.

TONELLI, D. et al. Efeito anestésico do midazolam intramuscular. Estudo clínico de 35 casos. Rev. Bras. Anestesiol., v. 41, p. 12, 1991. supl. 13

WAITZBERG, M.M; HOJAIJ, C.R. Considerações preliminares sobre o estudo da correlação ansiedade pós-operatório. Folha Médica., v. 90, n. 3, p. 97-103. 1985. 